Estudios Geológicos, 66(1)

enero-junio 2010, 13-23

ISSN: $0367-0449$

doi:10.3989/egeol.40105.087

\title{
Caracterización geoquímica y estudio comparativo de plagiogranitos de las Zonas Surportuguesa y Ossa-Morena (SO del Macizo Ibérico, España)

\author{
Geochemical characterization and comparative study \\ of plagiogranites from the Southportuguese and Ossa-Morena \\ Zones (SW Iberian Massif, Spain)
}

\author{
F. Bellido, A. Díez-Montes², T. Sánchez-García ${ }^{1}$
}

RESUMEN

En la Zona de Ossa-Morena (ZOM) y en la Zona Surportuguesa (ZSP) se encuentran plagiogranitos pertenecientes a asociaciones magmáticas y ambientes geodinámicos muy contrastados. Los primeros pertenecen a una asociación bimodal máfica-félsica relacionada con el rifting cámbrico-ordovícico y presentan bastantes afinidades con plagiogranitos oceánicos. Los segundos forman parte de una asociación volcánico-plutónica calcoalcalina carbonífera relacionada con la Orogenia Varisca. Ambos plagiogranitos son semejantes en diversos aspectos petrológicos y geoquímicos pero muestran diferencias en algunas relaciones de elementos inmóviles tales como $\mathrm{La} / \mathrm{Nb}$, Th/Nb, Th/La poco sensibles a los procesos de evolución magmática, metamorfismo y alteración y que son reflejo de la composición de las fuentes. Los plagiogranitos de la ZSP presentan una mayor homogeneidad composicional que los de la ZOM. Esto se interpreta como que los primeros tienen un origen cogenético, mientras que los segundos se derivan de diferentes fuentes (astenosféricas, litosféricas y crustales).

Palabras clave: Geoquímica, plagiogranitos, Zona de Ossa-Morena, Zona Surportuguesa.

\begin{abstract}
Plagiogranites and albitic rocks belonging to contrasted magmatic associations of different ages and geodynamic environments outcrop in Ossa-Morena (OMZ) and Surportuguese (SPZ) Zones. OMZ plagiogranites are related with Cambrian-Ordovician rifting and represent a part of the felsic members of a bimodal magmatic suite and show oceanic plagiogranites affinities. SPZ plagiogranites are related with a Carboniferous volcano-plutonic calc-alkaline suite related with Variscan Orogeny. Both plagiogranites show many similar petrological and geochemical characteristics but display some different immobile elements ratios as $\mathrm{La} / \mathrm{Nb}, \mathrm{Th} / \mathrm{Nb}, \mathrm{Th} / \mathrm{La}$ that are unaltered by metamorphism, magmatic differentiation and alteration processes and prove differences in their sources. SPZ plagiogranites show a more compositional homogeneity than $\mathrm{OMZ}$ ones. This is interpreted as a proof of cogenetic origin for SPZ plagiogranites and heterogeneous origin for $\mathrm{OMZ}$ plagiogranites, derived from the mix of different sources (astenospheric, lithospheric and crustal).
\end{abstract}

Key words: Geochemistry, plagiogranites, Ossa-Morena Zone, Southportuguese Zone.

\section{Introducción y contexto geológico}

En este trabajo se hace un estudio comparativo de plagiogranitos en dos zonas diferentes del Macizo Ibérico (Zonas Surportuguesa y Ossa-Morena) (figs. 1 y 2) y en dos contextos geodinámicos distintos. Uno de ellos corresponde a un evento extensional en el margen SW de Gondwana, que tuvo lugar durante el Rift Cámbrico Inferior-Ordovícico en Ossa-Morena y el otro está relacionado con una

\footnotetext{
1 IGME, C/ La Calera, 1, 28760-Tres Cantos, Madrid. Email: f.bellido@igme.es, t.sanchez@igme.es
}

2 IGME, C/ Azafranal, 48, 1. ${ }^{\circ}$ B. 37001 Salamanca. Email: al.diez@igme.es 


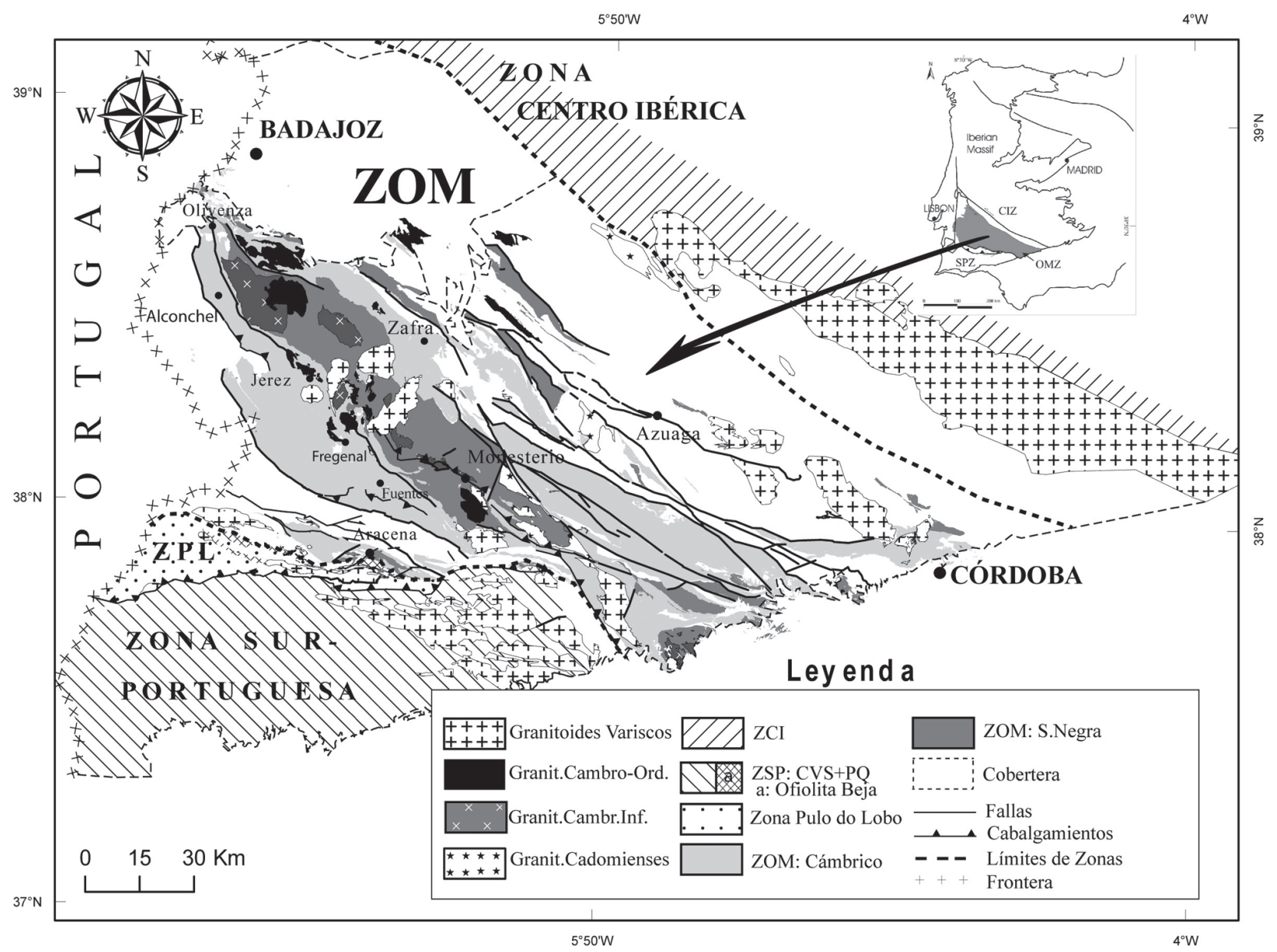

Fig. 1.-Esquema geológico de la zona de Ossa Morena.

colisión continental que tuvo lugar en la Zona Surportuguesa durante el Carbonífero en la Orogenia Varisca.

El término plagiogranito se utiliza en un sentido amplio, en el que se incluyen diversos tipos de rocas plutónicas y subvolcánicas ácidas, ricas en plagioclasa albítica.

En la ZOM (fig. 1) tras un proceso de acreción continental durante el Neoproterozoico, la evolución del margen norte de Gondwana comenzó en el Cámbrico Inferior con un levantamiento y erosión diferencial de antiguos segmentos del arco Cadomiense-Avaloniense (Quesada, 1991; Sánchez-García et al., 2003; Pereira et al., 2006, 2008; Murphy et al., 2006, entre otros). El comienzo de un proceso de extensión fue responsable del adelgazamiento cortical (Liñan \& Quesada, 1990), acompañado de un ascenso térmico en el manto que desencadenó importante actividad ígnea. En este evento magmático se han establecido dos períodos (Eventos Temprano y Principal) que culminaron con la apertura del Océano Rheico en el Ordovícico Inferior. Durante el evento Temprano se formaron complejos migmatíticos y estructuras core-complex en la parte media-superior de la corteza con generación de magmas félsicos peraluminosos a partir de fuentes crustales. El Evento Principal está representado por una asociación bimodal de rocas félsicas y máficas con escasas cantidades de rocas intermedias. Algunas rocas máficas tienen afinidades NMORB, mientras que otras presentan afinidades OIB o E-MORB. Las rocas ácidas e intermedias de este Evento Principal proceden de la evolución de mezclas de magmas crustales y mantélicos (Sánchez-García et al., 2003, 2008a, 2008b, 2010; Bellido et al., 2007), y es dentro de este Evento 


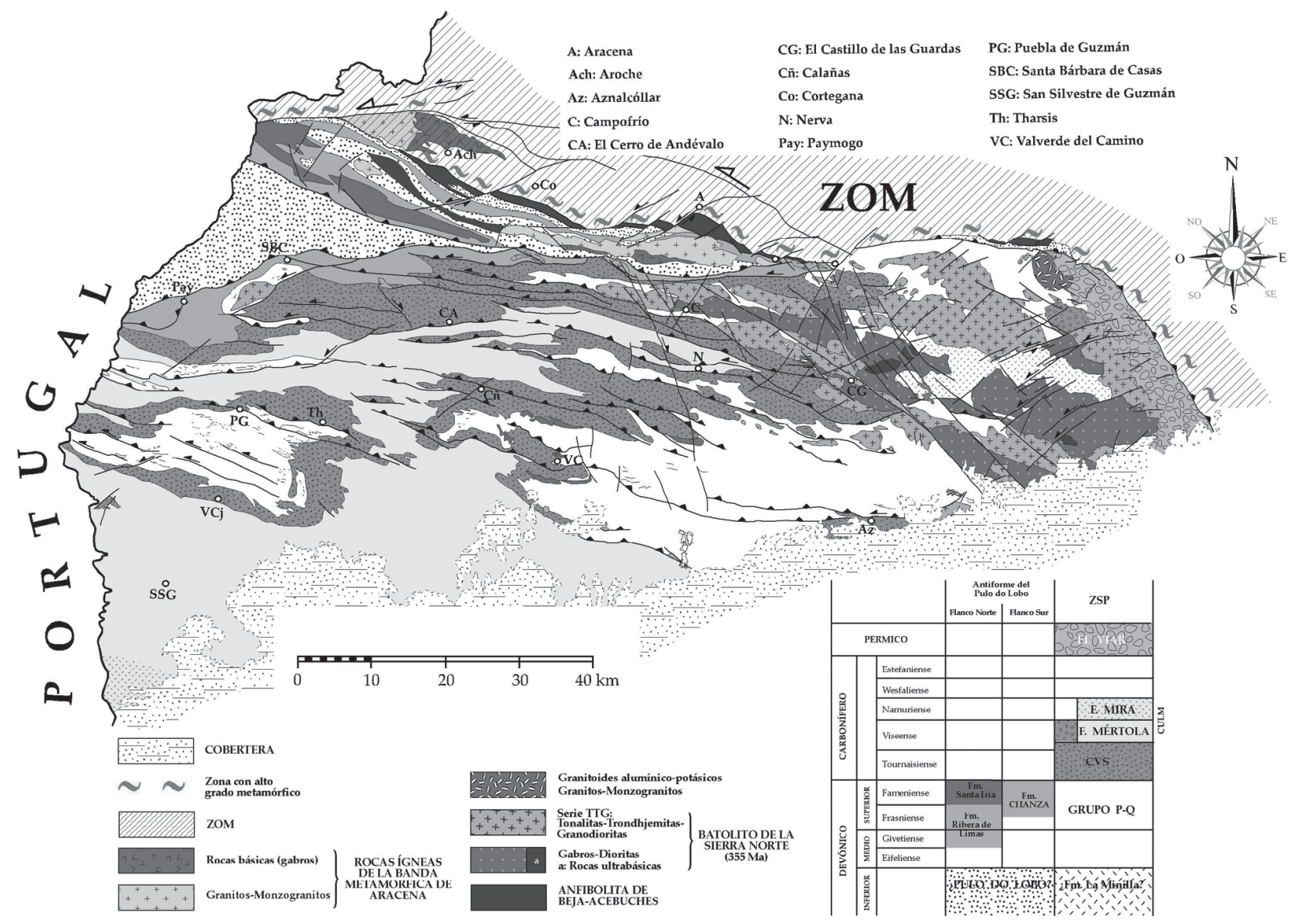

Fig. 2.-Esquema geológico de la Zona Surportuguesa.

Principal donde aparecen plagiogranitos relacionados con las rocas máficas. En este trabajo se han estudiado rocas del macizo de Feria y de las áreas de Jerez-Valle de Matamoros y de Fregenal-Fuentes de León (fig. 1). La edad de estas rocas está entre $517 \pm 2$ Ma del Granito de los Remedios y $502 \pm 2$ Ma de una riolita del área de Feria (Sánchez-García et al., 2008a).

En la ZSP (fig. 2) se desarrolla un importante magmatismo asociado a la evolución geodinámica de este sector de la Cadena Varisca que está representado por las rocas plutónicas del Batolito de la Sierra Norte (BSN) (De la Rosa, 1992), y por las rocas volcánicas del Complejo Vulcano-Sedimentario (CVS) de la Faja Pirítica Ibérica (FPI) (Munhá, 1983; Mitjavila et al., 1997).

El BSN está constituido principalmente por tres asociaciones magmáticas: a) Un conjunto de rocas básicas (gabros-dioritas), con algunos acumulados ultrabásicos. b) Una serie de intrusiones subvolcánicas de tonalitas, trondhjemitas y granodioritas con afinidad TTG. c) Un conjunto de granitoides alumínico-potásicos que forman plutones circunscritos que intruyen al resto de las rocas plutónicas y en los materiales plegados del CVS, produciendo metamorfismo de contacto sobre ellos (Díez-Montes \& Bellido Mulas, 2008).

Los granitoides de la asociación TTG presentan frecuentes zonas de mezcla con las rocas básicas (fig. 3), y su edad, de acuerdo con los datos radiométricos existentes, fluctúa entre $354,4+5 /-4 \mathrm{Ma}$ para el granito de Campofrío (Dunning et al., 2002) y 346,23 $\pm 0,81 \mathrm{Ma}$ para una tonalita cercana a Campofrío (Tucker Barrie et al., 2002).

El vulcanismo del CVS está representado esencialmente por una asociación calcoalcalina com- 

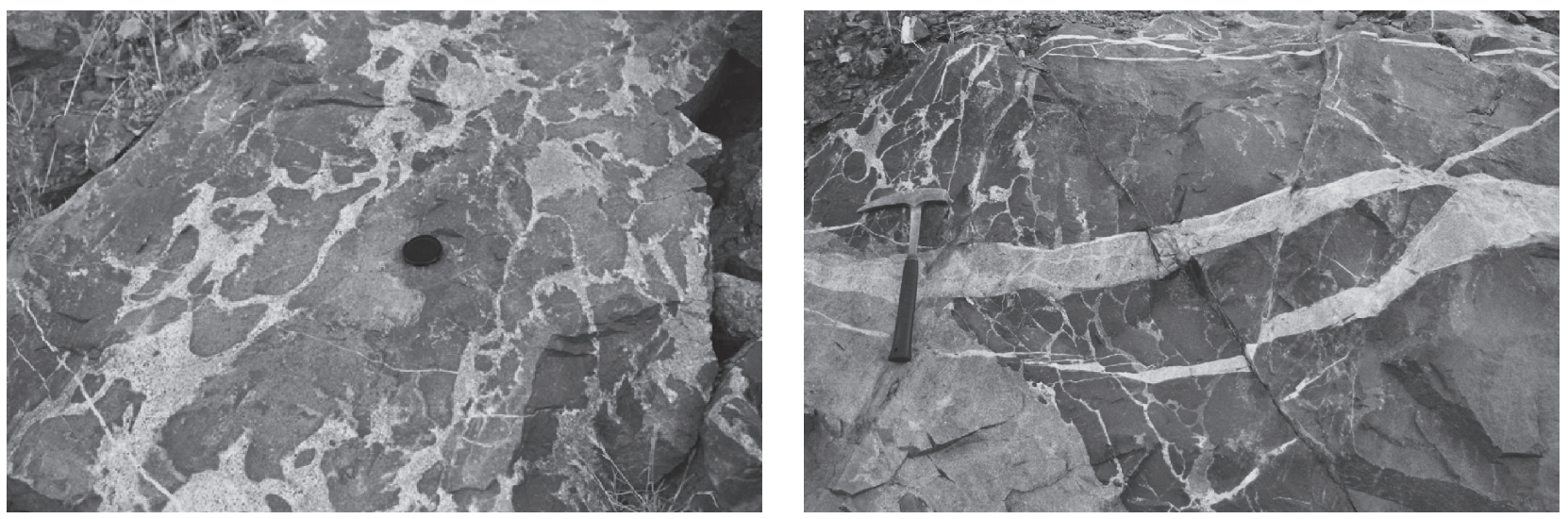

Fig. 3.-Detalle de las zonas de mezcla que hay en el contacto entre los plagiogranitos de la serie TTG y las rocas básicas (gabrosdioritas) que constituyen el Batolito de la Sierra Norte (BSN), dentro de la Zona Surportuguesa.

puesta por basaltos, andesitas basálticas, traquibasaltos, andesitas, dacitas y riolitas de edad Carbonífero Inferior.

\section{Petrología de los granitoides trondhjemíticos de la ZSP y de los plagiogranitos de la ZOM}

Los plagiogranitos estudiados en la ZOM son rocas que tienen colores blanquecinos o blancorosáceos, grano fino a medio y que con relativa frecuencia son porfídicos.

La mineralogía principal consta casi exclusivamente de cuarzo y plagioclasa albítica. Como minerales accesorios pueden encontrarse: biotita, anfíbol, feldespato potásico, circón, apatito magnetita, clinopiroxeno, fluorita y carbonatos. Entre los minerales secundarios aparecen clorita, sericita, moscovita, epidota, titanita, carbonatos y óxidos.

Las texturas más comunes son granudas hipidiomórficas de grano fino o porfídicas con fenocristales de albita y cuarzo y es relativamente frecuente la presencia de dominios granofídicos o micropegmatíticos intersticiales o generalizados (fig. 4). La deformación es variable, llegando a encontrarse albititas con texturas porfidoclásticas foliadas

Los materiales del macizo de Feria se caracterizan por ser más ricos en feldespatos, careciendo en general de fenocristales de cuarzo.

Los plagiogranitos de la ZSP tienen texturas que varían desde hipoabisales a plutónicas, y en general tienen un tamaño de grano medio a fino. Es muy característica la presencia de cristales de cuarzo. Su color en fresco es de gris claro a blanquecino, con tonalidades beige amarillentas cuando están alterados.
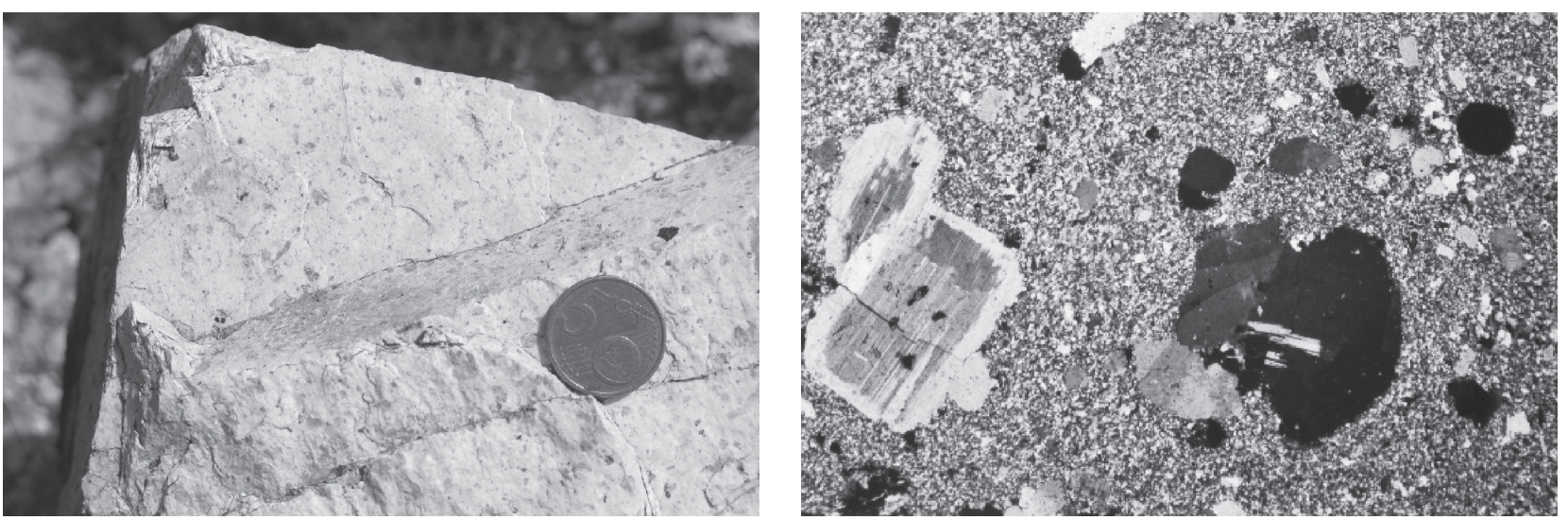

Fig. 4.-Aspecto textural de los pórfidos albíticos de La Berrona, en la Zona de Ossa-Morena. Aspecto microtextural de los pórfidos con fenocristales de albita y de cuarzo corroído con inclusiones de albita en una matriz félsica de grano fino (NC x 6.25). 

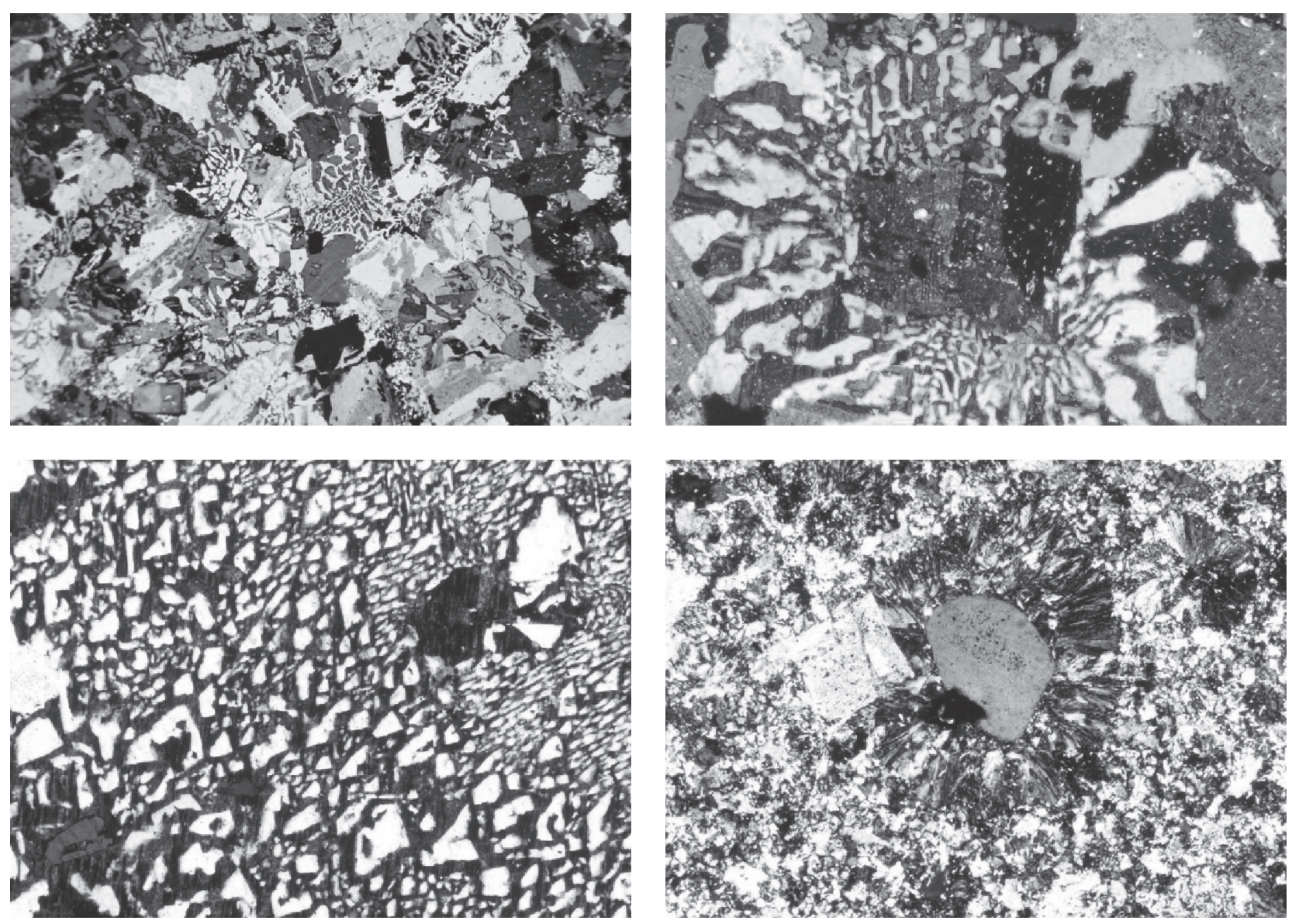

Fig. 5.-Aspectos microtexturales de los plagiogranitos pertenecientes a la serie TTG de la ZSP.

La mineralogía principal de estos plagiogranitos se compone de cuarzo, plagioclasa albítica, biotita, anfíbol, +/- feldespato potásico. Los minerales ferromagnesianos (principalmente biotita), se encuentran relegados, en general, a proporciones accesorias. Como minerales accesorios se encuentran opacos (ilmenita y sulfuros), titanita, apatito, circón y allanita. Entre los minerales secundarios aparecen sericita, clorita, epidota-clinozoisita, calcita, actinolita y feldespato potásico.

Con frecuencia presentan texturas hipidiomórficas con fenocristales dispersos y con dominios micrográficos que pueden ser muy abundantes, encontrándose en algunos casos texturas micrográfico-granofídicas generalizadas (fig. 5).

\section{Geoquímica}

El conjunto de materiales ígneos cámbricos al que pertenecen los plagiogranitos estudiados en la ZOM constituye una asociación bimodal compuesta esencialmente por rocas máficas y félsicas (fig. 6A), mientras que los materiales variscos de la ZSP constituyen una asociación ígnea calcoalcalina en la que se encuentra una proporción considerable de rocas intermedias (fig. 6B).

El estudio comparativo entre los plagiogranitos de ambas zonas se ha realizado a partir de rocas con $\mathrm{SiO}_{2}>67 \%$ y con relaciones $\mathrm{Na}_{2} \mathrm{O} / \mathrm{CaO}>4$, habiéndose seleccionado 22 muestras de la ZOM y 33 de la ZSP, cuyas composiciones medias figuran en la tabla 1.

El examen de las medias de ambos grupos de plagiogranitos pone de manifiesto que las de la ZSP son significativamente más ricas en $\mathrm{FeOt}, \mathrm{MnO}$, $\mathrm{MgO}$ y $\mathrm{K}_{2} \mathrm{O}$, mientras que las de la $\mathrm{ZOM}$ son más ricas en $\mathrm{TiO}_{2}, \mathrm{Na}_{2} \mathrm{O}$ y $\mathrm{P}_{2} \mathrm{O}_{5}$. También puede observarse que los plagiogranitos de la ZSP son algo más ricos en $\mathrm{SiO}_{2}$, lo que desde el punto de vista mineralógico se traduce en una mayor riqueza en cuarzo y una menor proporción de feldespatos. 
A ZOM
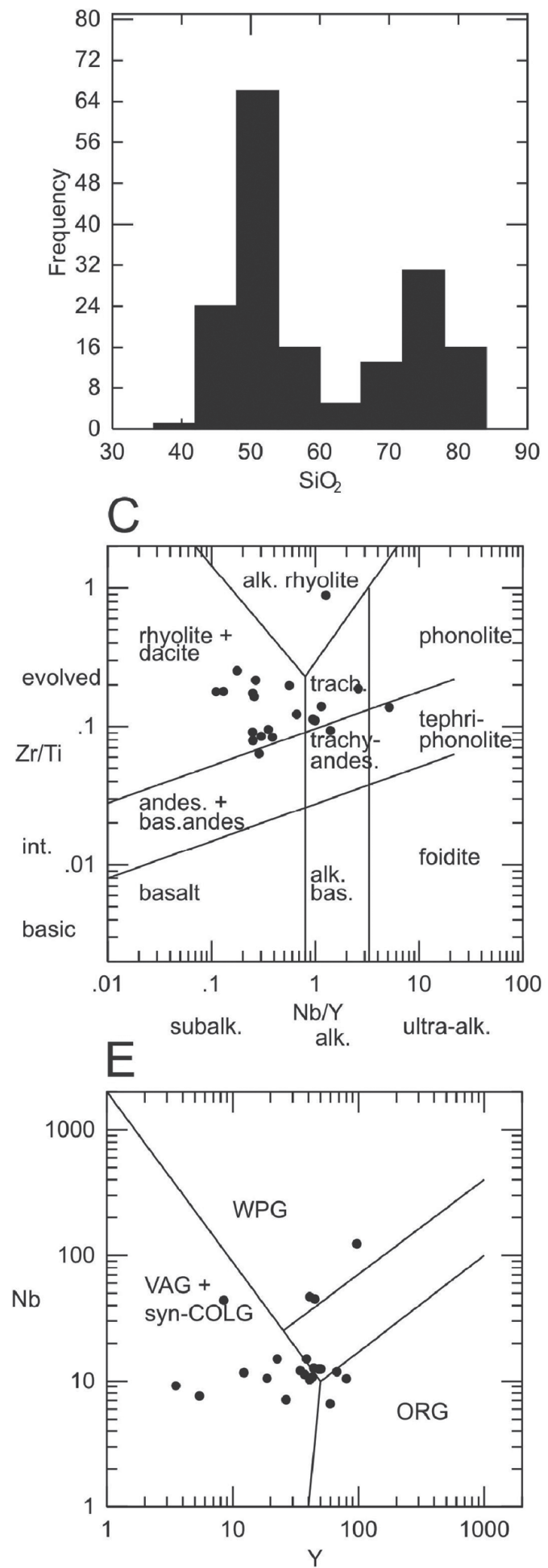
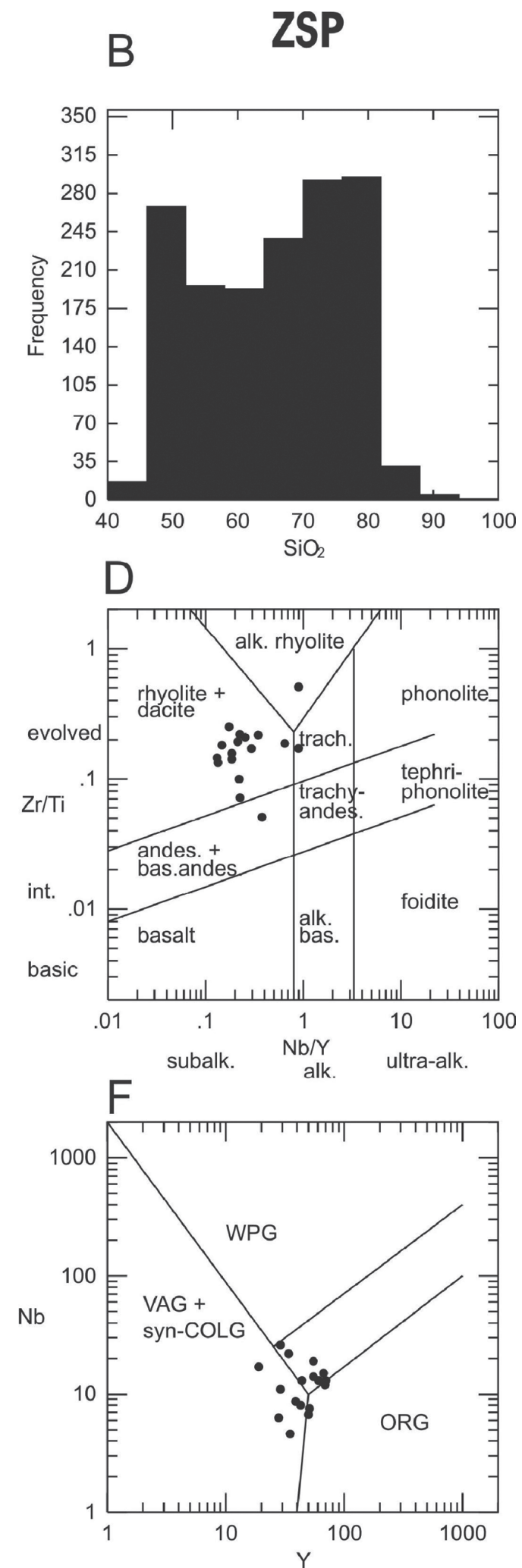

Fig. 6.-Características geoquímicas de la ZOM (columna izquierda) y de la ZSP (columna derecha). A) y $\mathrm{B}$ ) $\mathrm{Histogramas} \mathrm{de} \mathrm{SiO}_{2}$. C) y D) Clasificación petrológica según elementos inmóviles de Pearce (1996). E) y F) Caracterización geodinámica Y-Nb de Pearce et al. (1984). 
Tabla 1.-Composiciones medias de las rocas estudiadas en la ZOM y en la ZSP y del Manto Primitivo (MP) de Palme \& O’Neil (2004) y Corteza Continental Superior (CCS) de Rudnick \& Gao (2004)

\begin{tabular}{|c|c|c|c|c|c|c|c|c|c|}
\hline & $\mathrm{ZOM}$ & ZSP & MP & CCS & & $\mathrm{ZOM}$ & ZSP & M P & CCS \\
\hline $\mathrm{SiO}_{2}$ & 75,46 & 76,84 & 45,40 & 66,6 & $\mathrm{~Tb}$ & 1,02 & 1,19 & 0,105 & 0,7 \\
\hline $\mathrm{TiO}_{2}$ & 0,43 & 0,22 & 458 & 0,64 & Dy & 6,61 & 7,64 & 0,711 & 3,9 \\
\hline $\mathrm{Al}_{2} \mathrm{O}_{3}$ & 13,55 & 12,86 & 4,49 & 15,4 & Ho & 1,42 & 1,58 & 0,159 & 0,83 \\
\hline $\mathrm{FeOt}$ & 0,92 & 2,07 & 8,10 & 5,04 & Er & 4,39 & 4,99 & 0,465 & 2,3 \\
\hline $\mathrm{MnO}$ & 0,02 & 0,04 & 0,25 & 0,10 & $\mathrm{Tm}$ & 0,69 & 0,78 & 0,072 & 0,3 \\
\hline $\mathrm{MgO}$ & 0,33 & 0,67 & 36,77 & 2,48 & $\mathrm{Yb}$ & 4,53 & 4,65 & 0,462 & 2 \\
\hline $\mathrm{CaO}$ & 0,82 & 0,76 & 3,65 & 3,59 & $\mathrm{Lu}$ & 0,71 & 0,72 & 0,071 & 0,31 \\
\hline $\mathrm{Na}_{2} \mathrm{O}$ & 7,06 & 5,63 & 0,67 & 3,27 & Hf & 8,07 & 5,60 & 0,3 & 5,3 \\
\hline $\mathrm{K}_{2} \mathrm{O}$ & 0,38 & 0,88 & 0,06 & 2,80 & $\mathrm{Ta}$ & 2,16 & 0,70 & 0,04 & 0,9 \\
\hline \multirow[t]{2}{*}{$\mathrm{P}_{2} \mathrm{O}_{5}$} & 0,07 & 0,03 & 0,21 & 0,15 & $\mathrm{~Pb}$ & 4,75 & 15,00 & 0,185 & 17 \\
\hline & & & & & Th & 13,54 & 10,93 & 0,0834 & 10,5 \\
\hline $\mathrm{Cs}$ & 2,02 & 0,84 & 0,018 & 4,9 & $\mathrm{U}$ & 4,19 & 2,26 & 0,027 & 2,7 \\
\hline $\mathrm{Ga}$ & 21,22 & 17,07 & 4,4 & 17,5 & $\Sigma$ REE & 125,12 & 160,72 & 8,278 & 149,14 \\
\hline $\mathrm{Ge}$ & 1,11 & 1,60 & 1,2 & 1,4 & & & & & \\
\hline $\mathbf{R b}$ & 11,98 & 34,64 & 0,605 & 84 & $\mathrm{La} / \mathrm{Nb}$ & 1,36 & 2,86 & 1,17 & 2,58 \\
\hline $\mathrm{Sr}$ & 83,00 & 76,89 & 20,3 & 320 & $\mathrm{Th} / \mathrm{Nb}$ & 1,12 & 0,89 & 0,14 & 0,88 \\
\hline $\mathrm{Y}$ & 38,42 & 41,56 & 10,81 & 21 & $\mathrm{Zr} / \mathrm{Nb}$ & 23,05 & 17,90 & 18,38 & 16,08 \\
\hline $\mathrm{Zr}$ & 330,81 & 197,32 & 10,81 & 193 & $\mathrm{Zr} / \mathrm{Th}$ & 61,12 & 20,07 & 129,62 & 18,38 \\
\hline $\mathrm{Nb}$ & 21,28 & 12,75 & 0,588 & 12 & $\mathrm{Zr} / \mathrm{Hf}$ & 42,47 & 33,74 & 36,03 & 36,42 \\
\hline $\mathrm{Ba}$ & 82,50 & 143,93 & 6,75 & 628 & $\mathrm{Th} / \mathrm{La}$ & 1,13 & 0,32 & 0,12 & 0,34 \\
\hline $\mathrm{La}$ & 21,22 & 27,90 & 0,686 & 31 & $\mathrm{Ba} / \mathrm{La}$ & 4,17 & 7,06 & 9,84 & 20,26 \\
\hline $\mathrm{Ce}$ & 46,77 & 58,53 & 1,786 & 63 & $\mathrm{~K} / \mathbf{R b}$ & 218 & 245 & 430 & 277 \\
\hline $\mathrm{Pr}$ & 5,49 & 7,59 & 0,270 & 7,1 & $\mathrm{Ca} / \mathrm{Sr}$ & 107 & 74 & 1286 & 80 \\
\hline $\mathrm{Nd}$ & 21,27 & 30,48 & 1,327 & 27 & $\mathrm{Ba} / \mathrm{Sr}$ & 0,74 & 2,45 & 0,33 & 1,96 \\
\hline Sm & 4,83 & 6,73 & 0,431 & 4,7 & $\mathrm{Th} / \mathrm{U}$ & 4,68 & 4,27 & 3,09 & 3,89 \\
\hline $\mathrm{Eu}$ & 0,80 & 0,86 & 0,162 & 1 & $\mathrm{Nb} / \mathrm{Ta}$ & 9,85 & 18,21 & 14,7 & 13,4 \\
\hline Gd & 5,37 & 7,08 & 0,571 & 4 & $\mathrm{La} / \mathrm{Yb}_{\mathrm{n}}$ & 3,15 & 4,04 & 1 & 10,44 \\
\hline
\end{tabular}

Utilizando los criterios de Barker (1979) basados en las proporciones normativas de Ab, An y Or, todos los plagiogranitos estudiados son clasificables como trondhjemitas.

Con respecto al grado de saturación en $\mathrm{Al}_{2} \mathrm{O}_{3}$, la mayoría de los plagiogranitos de ambas zonas son peraluminosos, aunque la presencia de tipos subaluminosos es más común entre los de la ZOM.

La clasificación realizada a partir de elementos inmóviles, $\mathrm{Nb} / \mathrm{Y}-\mathrm{Zr} / \mathrm{Ti}$ (Pearce, 1996), para evitar los efectos de la alteración y el metamorfismo, pone de manifiesto que la mayoría de los plagiogranitos de la ZOM (fig. 6C) tienen composiciones que corresponden a riolitas y riodacitas subalcalinas, aunque algunos de ellos se proyectan en campos de rocas alcalinas e incluso ultra-alcalinas. Entre las muestras de la ZSP (fig. 6D) son muy escasas las de afinidad alcalina.

Con respecto a los elementos del grupo de las tierras raras, los plagiogranitos de la ZOM presentan unos contenidos medios $\Sigma$ REE $=125,12$ con unas relaciones medias $(\mathrm{La} / \mathrm{Yb})_{\mathrm{n}}=3,15$, mientras que en los plagiogranitos de la ZSP, el contenido medio en tierras raras y su fraccionamiento son superiores $\left(\Sigma \mathrm{REE}=160,72\right.$ y $\left.(\mathrm{La} / \mathrm{Yb})_{\mathrm{n}}=4,04\right)$.

En los diagramas de REE normalizados a la composición del manto primitivo (Palme \& O'Neil, 2004; figs. 7A y 7B), puede verse que los plagiogranitos cámbricos de la ZOM, presentan una apreciable dispersión de espectros. Los tipos más comunes presentan un moderado fraccionamiento de las LREE y tramos correspondientes a las HREE bastante planos, con anomalías negativas de Eu bastante acentuadas. No obstante, también se encuentran algunas muestras con espectros bastante rectilíneos y fraccionamientos similares para las LREE y las HREE, y otras con empobrecimientos de las tierras raras más ligeras. Por el contrario, los plagiogranitos de la ZSP, presentan unos espectros de REE bastante homogéneos, con fraccionamientos de LREE moderados, escaso fraccionamiento de las HREE y apreciables anomalías negativas de Eu.

En los diagramas Spider normalizados a la composición del manto primitivo de Palme \& O'Neil (2004; figs. 7C y D), se observa que un grupo mayoritario de los plagiogranitos de la ZOM presenta 

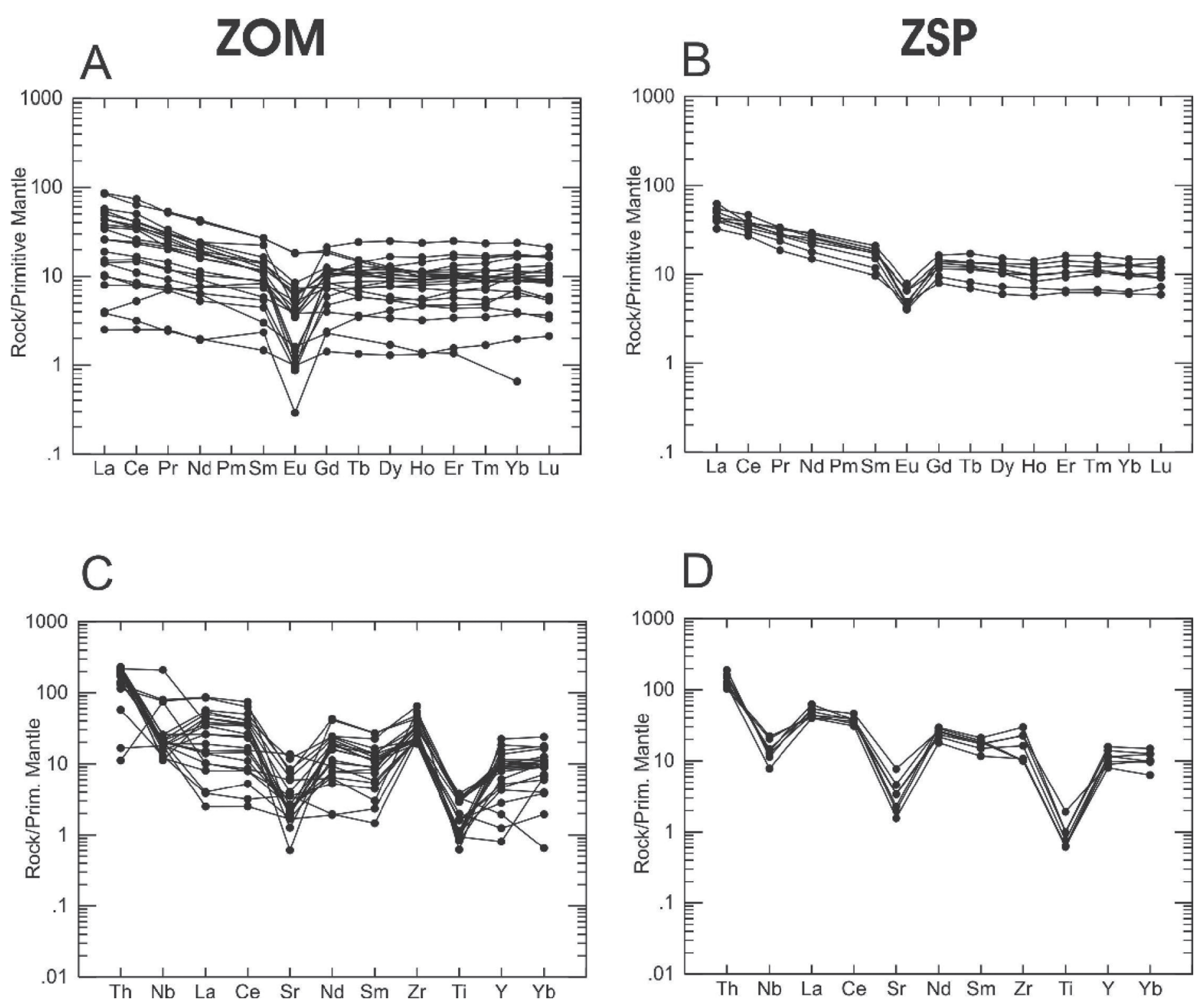

Fig. 7.-A) y B) Modelo de REE normalizado al Manto Primitivo de Palme \& O'Neil (2004) de la ZOM (columna izquierda) y de la ZSP (columna derecha). C) y D) Diagrama spider normalizado al Manto Primitivo de Palme \& O'Neil (2004).

anomalías negativas de $\mathrm{Nb}, \mathrm{Sr}$ y $\mathrm{Ti}$, y positivas de Th y Zr. No se observan anomalías negativas de $\mathrm{Nb}$ en las muestras del macizo de Feria y de un macizo situado en las inmediaciones de Fuentes de León. Por otra parte, se observan contenidos bajos de Th e $\mathrm{Y}$ en los plagiogranitos de los Remedios y Fregenal.

Los plagiogranitos de la ZSP presentan unos espectros similares a los de la ZOM, con anomalías negativas generalizadas de $\mathrm{Nb}, \mathrm{Sr}$ y $\mathrm{Ti}$ y positivas de $\mathrm{Th}$ y $\mathrm{Zr}$. En este caso, las pautas son más homogéneas.

También es patente una mayor riqueza de $\mathrm{Zr}$ en los plagiogranitos de la ZOM (tabla 1), que tienen unos contenidos medios de 331 ppm contra 197 ppm en los plagiogranitos de la ZSP.
El examen de las relaciones elementales de los elementos inmóviles más incompatibles y que están menos afectadas por los procesos de fraccionamiento, metamorfismo y alteración $(\mathrm{La} / \mathrm{Nb}, \mathrm{Th} / \mathrm{Nb}$, $\mathrm{Th} / \mathrm{La}$ y $\mathrm{Zr} / \mathrm{Th}$; tabla 1), pone de manifiesto que los valores de estas relaciones en los plagiogranitos de la ZSP son bastante semejantes a los de la Corteza Continental Superior (CCS) (Rudnick \& Gao, 2004), siendo, a su vez, sensiblemente diferentes a los valores medios de los plagiogranitos de la ZOM.

En cuanto a la caracterización geodinámica de los materiales estudiados, en los diagramas $\mathrm{Y}-\mathrm{Nb}$ de Pearce et al. (1984; figs. 6E y F), las muestras de la ZOM presentan bastante dispersión, y aunque se 
proyectan principalmente en el campo orogénico (VAG + syn-COLG), hay algunas muestras que también se sitúan en los campos de los granitos oceánicos (ORG), o de los de dorsales anómalas, o intraplaca (WPG). Los plagiogranitos de la ZSP presentan una dispersión menor, proyectándose todos ellos en una nube situada en la divisoria entre los campos de los granitoides orogénicos y los de dorsales anómalas. Estos resultados son en buena parte incongruentes con la realidad geológica, ya que todos los materiales cámbricos estudiados en la ZOM son de carácter anorogénico, mientras que los plagiogranitos de la ZSP se asocian a un evento magmático sinorogénico varisco.

\section{Discusión y conclusiones}

Los plagiogranitos cámbricos estudiados en la $\mathrm{ZOM}$ corresponden en su mayoría a trondhjemitas bajas en $\mathrm{Al}_{2} \mathrm{O}_{3}$ y ricas en $\mathrm{Yb}\left(\mathrm{Al}_{2} \mathrm{O}_{3}<14,5 \%\right.$ e $\mathrm{Yb}>1)$, que serían de afinidad oceánica según Arth (1979); no obstante, se encuentran entre ellos algunos tipos más ricos en $\mathrm{Al}_{2} \mathrm{O}_{3}$, aunque también con contenidos en $\mathrm{Yb}$ elevados. En el caso de los plagiogranitos de la ZSP, todas las muestras consideradas son identificables con trondhjemitas pobres en $\mathrm{Al}_{2} \mathrm{O}_{3}$ y ricas en $\mathrm{Yb}$.

Las trondhjemitas pobres en $\mathrm{Al}_{2} \mathrm{O}_{3}$ suelen tener espectros de REE, normalizados al condrito, planos o débilmente fraccionados, con anomalías negativas de Eu y contenidos en HREE relativamente elevados. No obstante, en el caso de las muestras estudiadas, la mayoría de los espectros tienen un fraccionamiento moderado de las LREE, con anomalías negativas de Eu y fraccionamientos de HREE muy débiles o inapreciables. Sólo se encuentran muestras con espectros planos o empobrecidos en LREE entre los plagiogranitos de la ZOM.

La relativa homogeneidad que presentan los plagiogranitos variscos de la ZSP se interpreta que es debida a una procedencia común a partir de la diferenciación de magmas de una asociación plutónica TTG esencialmente monogenética (rocas plutónicas del BSN).

En el caso de los plagiogranitos de la ZOM el hecho de que puedan pertenecer en algunos casos a asociaciones alcalinas (Feria), o en otros procedan de la interacción de magmas basálticos de distintos tipos (E-MORB y N-MORB) con distintas proporciones de materiales corticales, determina que sus características geoquímicas sean más heterogéneas.
Aunque los plagiogranitos y las albititas de la ZOM y de la ZSP presentan bastantes analogías mineralógicas o composicionales, tienen sensibles diferencias en algunas relaciones elementales como $\mathrm{La} / \mathrm{Nb}, \mathrm{Th} / \mathrm{Nb}$ y $\mathrm{Th} / \mathrm{La}$, que están poco afectadas por los procesos petrogenéticos y que son un reflejo de la composición de los materiales de las áreas fuente. Estas relaciones son relativamente similares en los materiales de la ZSP a las de la CCS (Rudnick \& Gao, 2004) y de series calcoalcalinas. Por el contrario, los materiales de la ZOM tienen relaciones más dispares, debidas a unos contenidos de Th relativamente altos.

Las incongruencias en la caracterización geodinámica de estos plagiogranitos a partir de criterios geoquímicos es interpretada como el reflejo de la complejidad de su génesis, en la que intervienen componentes mantélicos y corticales, y en que los parámetros geoquímicos que influyen en esta caracterización geodinámica sean, en parte, heredados de los materiales originarios.

Asociaciones trondhjemíticas pobres en $\mathrm{Al}_{2} \mathrm{O}_{3}$ como las de la ZSP y de las que derivarían los plagiogranitos estudiados en esta zona, se pueden formar por cristalización fraccionada de basaltos o por fusión parcial de anfibolitas a bajas presiones, con residuos con plagioclasa, clinopiroxeno y sin granate.

Los plagiogranitos de la ZOM deben haberse formado en bastantes casos por evolución de magmas basálticos N-MORB o E-MORB que han asimilado distintas proporciones de componentes corticales, aunque en otros procederían de la diferenciación de magmas básicos alcalinos.

Resultados provisionales de estudios isotópicos Sm-Nd que se están realizando en los materiales de la ZSP y datos inéditos sobre plagiogranitos de la ZOM (Carriedo, 2009, com. pers.) revelan valores negativos bajos de $\varepsilon \mathrm{Nd}$, lo que pone de manifiesto la participación de materiales corticales y mantélicos en su génesis.

Las pautas generales de variación de las REE en la mayoría de los plagiogranitos de ambas zonas, sin empobrecimientos de las HREE y con sensibles anomalías negativas de Eu, apoyan procesos petrogenéticos a bajas presiones, con fraccionamiento de plagioclasa y sin intervención de granate. La presencia de anomalías negativas de Sr estaría justificada por el fraccionamiento de plagioclasa, mientras que las anomalías negativas de $\mathrm{Nb}$ y Ti pueden ser debidas a cristalización de anfíbol o a la presencia de minerales titanados $(\mathrm{Ru})$ en los residuos de fusión. Los enriquecimientos en LREE y Th que se observan en gran parte de los materiales estudiados son debidos a 
la participación de componentes corticales en la génesis de estos plagiogranitos, lo que también es apoyado por los datos isotópicos provisionales.

Los plagiogranitos de la ZSP pertenecen a una asociación plutónica TTG relacionada con un vulcanismo asociado a importantes depósitos de sulfuros masivos en la Faja Pirítica Ibérica (Díez Montes \& Bellido Mulas, 2008), mientras que los plagiogranitos de la ZOM están relacionados con un magmatismo bimodal cámbrico (Sánchez-García et al., 2003, 2008a, 2010) y en algunos casos se encuentran yacimientos de Fe de afinidad IOCG asociados a ellos (Carriedo \& Tornos, 2006), por lo que el estudio detallado de estos materiales plutónicos puede constituir un importante apoyo para la investigación y discriminación de los procesos magmáticos que influyen en la formación de estos tipos de yacimientos.

\section{AGRADECIMIENTOS}

Este trabajo ha sido realizado en el marco de los siguientes proyectos: Proyecto no 348 (Neoproterozoico-Cámbrico ZOM), financiado por el IGME, Proyecto n⿳o CGL2006-12245BTE del Ministerio de Educación y ciencia, y proyecto FEDER, no CGL2006-0378.

\section{Referencias}

Arth, J.G. (1979). Some trace elements in trondhjemites. Their implications to magma genesis and paleotectonic setting. In: Trondhjemites, dacites and related rocks (Barker, F., ed.). Developments in Petrology (16). Elsevier, 123-132.

Barker, F. (1979). Trondhjemite: Definition, environment and hypothesis of origin. In: Trondhjemites, dacites and related rocks (Barker, F., ed.). Developments in Petrology (16). Elsevier, 1-12.

Bellido Mulas, F.; Sánchez-García, T. \& Díez Montes, A. (2007). Caracterización petrológica y geoquímica de los materiales volcánicos y volcanosedimentarios de las capas de Bodonal-Cala (Zona de Ossa-Morena, SW de España). VI Congreso Ibérico de Geoquímica, Vila Real (Portugal). Extended abstract, 93-96.

Carriedo, J. \& Tornos, F. (2006). Los yacimientos de óxidos de hierro y mineralizaciones de cobre-oro asociadas del SO peninsular: un modelo vertical de evolución. Macla, 6: 135-136.

De la Rosa, J.D. (1992). Petrología de las rocas básicas y granitoides del Batolito de la Sierra Norte de Sevilla. Zona Surportuguesa. Macizo Ibérico. Tesis Doctoral, Universidad de Sevilla, 312 pp. y anexos.

Díez Montes, A. \& Bellido Mulas, F. (2008). Magmatismos TTG y Al-K en la Zona Surportuguesa. Relaciones entre plutonismo y vulcanismo. Geo-Temas, 10: 1449-1452.
Dunning, G.R.; Díez Montes, A.; Matas, J.; Martín Parra, L.M.; Almarza, J. \& Donaire, M. (2002). Geocronología U/Pb del volcanismo ácido y granitoides de la Faja Pirítica Ibérica (Zona Surportuguesa). Geogaceta, 32: 127-130.

Liñán, E. \& Quesada, C. (1990). Stratigraphy: Rift Phase (Cambrian). In: Pre-Mesozoic Geology of Iberia (Dallmeyer, R.D. \& Martínez García, E., eds.). SpringerVerlag, Berlin, 259-266.

Mitjavila, J.; Martí, J. \& Soriano, C. (1997). Magmatic evolution and tectonics setting of the Iberian Pyrite Belt Volcanism. Journal of Petrology, 38: 727-755. doi:10.1093/petrology/38.6.727

Munhá, J. (1983). Hercinian magmatism in the Iberian Pyrite Belt. In: The Carboniferous of Portugal (Lemos de Souza, J. \& Oliveira, J.T., eds.). Memorias Serviços Geologicos de Portugal, 29: 39-81.

Murphy, J.B.; Gutiérrez-Alonso, G.; Nance, R.D.; Fernández-Suárez, J.; Keppie, J.D.; Quesada, C.; Strachan, R.A. \& Dostal, J. (2006). Origin of the Rheic Ocean: Rifting along a Neoproterozoic suture? Geology, 34: 325-328. doi:10.1130/G22068.1

Palme, H. \& O'Neill, H.S.C. (2004). Cosmochemical estimates of mantle composition. In: Treatise on Geochemistry, Volume 2: The mantle and core (Carlson, R.W., ed. and Holland, H.D. \& Turekian, K.K., exec. eds.), Elsevier-Pergamon, Oxford, 1-38. doi:10.1016/B0-08-043751-6/02177-0

Pearce, J.A. (1996). A user's guide to basalt discrimination diagrams. In: Trace element geochemistry of volcanic rocks; applications for massive sulphide exploration (Bailes, A.H.; Christiansen, E.H.; Galley, A.G.; Jenner, G.A.; Keith, J.D.; Kerrich, R.; Lentz, D.R.; Lesher, C.M.; Lucas, S.B.; Ludden, J.N.; Pearce, J.A.; Peloquin, S.A.; Stern, R.A.; Stone, W.E.; Syme, E.C.; Swinden, H.S. \& Wyman, D.A., eds.). Short Course Notes - Geological Association of Canada, 12: 79-113.

Pearce, J.A.; Harris, N.B.W. \& Tindle, A.G. (1984). Trace element discrimination diagrams for the tectonic interpretation of granitic rocks. Journal of Petrology, 25: 956-983.

Pereira, M.F.; Chichorro, M.; Linnemann, U.; Eguiluz, L. \& Silva, J.B. (2006). Inherited arc signature in Ediacaran and Early Cambrian basins of the Ossa-Morena Zone (Iberian Massif, Portugal): Paleogeographic link with European and North African Cadomian correlatives. Precambrian Research, 144: 297-315. doi:10.1016/j.precamres.2005.11.011

Pereira, M.F.; Chichorro, M.; Williams, I.S. \& Silva, J.B. (2008). Zircon U-Pb geochronology of paragneisses and biotite granites from the SW Iberian Massif (Portugal): evidence for a palaeogeographical link between the Ossa-Morena Ediacaran basins and the West African craton. In: The boundaries of the West African Craton (Ennih, N. \& Liegeois, J.P., eds.). Geological Society of London Special Publication, 297: 385-408.

Quesada, C. (1991). Geological constraints on the Paleozoic tectonic evolution of tectonostratigraphic terranes in the Iberian Massif. Tectonophysics 185: 225-245. doi:10.1016/0040-1951(91)90446-Y 
Rudnick, R.L. \& Gao, S. (2004). Composition of the Continental Crust. In: Treatise on Geochemistry, Vol. 3: The crust (Rudnick, R.L., ed. and Holland, H.D. \& Turekian, K.K., exec. eds.), Elsevier-Pergamon, Oxford, 1-64.

Sánchez-García, T.; Bellido, F. \& Quesada, C. (2003). Geodynamic setting and geochemical signatures of Cambrian-Ordovician rift-related igneous rocks (Ossa-Morena Zone, SW Iberia). Tectonophysics, 365: 233-255. doi:10.1016/S0040-1951(03)00024-6

Sánchez-García, T.; Quesada, C.; Bellido, F.; Dunning, G. \& González de Tanago, J. (2008a). Two-step magma flooding of the upper crust during rifting: The Early Paleozoic of the Ossa Morena Zone (SW Iberia). Tectonophysics, 461: 72-90. doi:10.1016/j.tecto.2008.03.006

Sánchez-García, T.; Bellido, F.; Pereira, M.F.; LópezGuijarro, R.; Quesada, C.; Chichorro, M.; Silva, J.B. \&
Pin, Ch. (2008b). Expresión magmática temprana de un rift intracontinental en el margen de Gondwana durante el Cámbrico inferior: Zona de Ossa-Morena (SW del Macizo Ibérico, Portugal, España). GeoTemas, 10: 1567-1572.

Sánchez-García, T.; Bellido, F.; Pereira, M.F.; Chichorro, M.; Quesada, C.; Pin, Ch. \& Silva, J.B. (2010). Rift-related volcanism predating the birth of the Rheic Ocean (Ossa-Morena Zone, SW Iberia). Gondwana Research, 17: 392-407. doi:10.1016/j.gr.2009.10.005

Tucker Barrie, C.; Amelin, Y. \& Pascual, E. (2002). U-Pb Geochronology of VMS mineralization in the Iberian Pyrite Belt. Mineralium Deposita, 37: 684-703. doi:10.1007/s00126-002-0302-7

Recibido el 19 de octubre de 2009 Aceptado el 15 de diciembre de 2009 Publicado online el 22 de abril de 2010 